\title{
Neutron Detection Performance of Silicon Carbide and Diamond Detectors with Incomplete Charge Collection Properties ${ }^{\text {年. }}$.
}

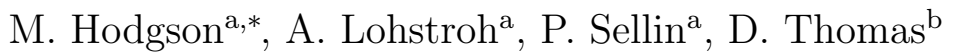 \\ ${ }^{a}$ Department of Physics, University of Surrey, Guildford, GU2 7XH, United Kingdom \\ ${ }^{b} N P L^{\odot}$, Teddington, TW11 OLW, United Kingdom
}

\begin{abstract}
The benefits of neutron detection and spectroscopy with carbon based, wide band gap, semiconductor detectors has previously been discussed within literature. However, at the time of writing there are still limitations with these detectors related to availability, cost, size and perceived quality. This study demonstrates that lower quality materials - indicated by lower charge collection efficiency (CCE), poor resolution and polarisation effect - available at wafer scale and lower cost, can fulfil requirements for fast neutron detection and spectroscopy for fluxes over several orders of magnitude, where only coarse energy discrimination is required.

In this study, a single crystal diamond detector (D-SC, with 100\% CCE), a polycrystalline diamond (D-PC, with $\approx 4 \% \mathrm{CCE}$ ) and semi-insulating silicon carbide (SiC-SI, with $\approx 35 \% \mathrm{CCE}$ ) have been compared for alpha and fast neutron performance.

All detectors demonstrated alpha induced polarisation effects in the form of a change of both energy peak position and count rate with irradiation time. Despite these operational issues the ability to detect fast neutrons and distinguish neutron energies was observed.

This performance was demonstrated over a wide dynamic range (500 40,000 neutrons/s), with neutron induced polarisation being demonstrated in D-PC and SiC-SI at high fluxes.
\end{abstract}

\footnotetext{
(C)British Crown Owned Copyright 2016/AWE.

*Corresponding author

Email address: michael.hodgson@becq.co.uk (M. Hodgson)
} 
Keywords:

Semi-Insulating Silicon Carbide, Single Crystal Diamond, Polycrystalline Diamond, Neutron Detection, Polarization, Semiconductor Radiation

Detectors.

\section{Introduction}

Carbon based semiconductor detectors such as silicon carbide ( $\mathrm{SiC})$ and diamond (D) offer a number of advantages for fast neutron detection purposes. The low atomic number $(Z)$ of carbon means that the maximum amount of energy transferable through elastic scattering with incident neutrons is $28 \%$ [1] allowing for direct fast neutron detection within these materials, removing manufacturing and efficiency issues associated with conversion layers [2].

Furthermore the low $Z$ number ensures that $\mathrm{SiC}$ and $\mathrm{D}$ are less sensitive to photon radiation relative to other semiconductor detectors. The result would be a reduced neutron-gamma cross-sensitivity in mixed radiation fields, which is a particularly important feature in most neutron environments.

However, although the use of $\mathrm{SiC}$ and $\mathrm{D}$ semiconductors for radiological detection applications was demonstrated as far back as the 1950's [3][4], they are still considered a relatively immature detector technology. In fact, it wasn't until a resurgence in the 1990's, driven by the need for electronics which could withstand high temperatures and radiation doses, that high quality, low defect material started to become available. As such there are only a handful of commercial suppliers in the world that grow $\mathrm{SiC}$ and $\mathrm{D}$ for detection applications. This, combined with the complexity of fabricating these materials, means that the cost remains relatively high, with good quality epitaxial silicon carbide ( $\mathrm{SiC}-\mathrm{EP}$ ) and electronic grade single crystal diamond (D-SC) being in the order of 175 times and 5000 times more expensive than silicon PIN detectors per $\mathrm{mm}^{2}$ (2014 wafer costs).

Cheaper, lower grade material is available in the form of semi-insulating silicon carbide (SiC-SI) and polycrystalline diamond (D-PC), 30 times and 170 times more expensive than silicon PIN per $\mathrm{mm}^{2}$ respectively, but these detectors generally demonstrate incomplete charge collection characteristics and relatively poor resolution.

With the exception of SiC-EP, all these detectors have also demonstrated some form of the so-called polarisation effect during irradiation, that being a change in the acquired spectrum and / or count rate with time [5][6][7]. This 
particular effect is prevalent in low doped, wide band gap semiconductors and is a result of charge carriers being trapped for long periods of time, leading to a change in the space charge distribution.

Despite these issues, the ability to directly detect neutrons has previously been demonstrated in SiC-SI [8], D-SC [9] and D-PC [10], among others. Work has now been conducted to quantify and compare the effect of incomplete charge collection, poor energy resolution and polarisation effects on the neutron detection capabilities for each of these detectors. This demonstrates that suitable neutron detection performance and stability is possible with all the detectors tested, increasing the options available for practical applications where large areas/volumes are needed.

\section{Theory}

\section{Semiconductor Detectors}

In practical semiconductor detectors, defects and impurities may be introduced during growth, fabrication and operation. These factors may introduce traps within the band gap region of a semiconductor's electronic structure which act to capture the created charge carriers (electrons or holes) and immobilise them for a period of time or even neutralise them completely.

These traps exist at specific energy levels $\left(E_{t}\right)$ within the band gap region, which the trapped charge carriers must subsequently overcome in order to once again freely move through the material. The trap energy level $\left(E_{t}\right)$ for specific carrier traps is referred to relative to the band energy, so either the conduction energy band $\left(-E_{c}\right)$ for electron trapping or the valance band $\left(+E_{v}\right)$ for hole trapping.

As given by Lutz [11], the average emission time of a trapped charged carrier, or the detrapping time, $\left(t_{t}\right)$ is dependent upon both the temperature $(T)$ and the energetic location of the trap within a specific material. Therefore shallow traps are quite close to the allowed energy bands and charge carriers tend to migrate between the energy levels quickly. However, deep traps tend to exist near the mid-point of the forbidden region and as such the amount of energy required for the trapped carriers to migrate back to the allowed energy band may be large [1][12]. This therefore may result in quite long periods of immobilisation, especially within wide band gap semiconductors where the mid-point energy may be larger than even the band gap of standard semiconductors like silicon, as illustrated in Table 1. 


\begin{tabular}{|c|c|c|c|}
\hline$E_{t}(\mathrm{eV})$ & $\sigma\left(\mathrm{cm}^{2}\right)$ & $t_{t}($ at $300 \mathrm{~K})$ & Reference \\
\hline 0.31 & $3 \times 10^{-16}$ & $0.5 \mathrm{~ms}$ & {$[13]$} \\
\hline 0.38 & $3 \times 10^{-16}$ & - & {$[14]$} \\
\hline 0.39 & $3.2 \times 10^{-19}$ & $13 \mathrm{~ms}$ & {$[15]$} \\
\hline 1.14 & $9.5 \times 10^{-14}$ & $\approx 13 \mathrm{hrs}$ & {$[15]$} \\
\hline 1.23 & $4 \times 10^{-13}$ & $\approx 76$ days & {$[15]$} \\
\hline 1.86 & - & $\approx \times 10^{9} \mathrm{yr}$ & {$[16]$} \\
\hline
\end{tabular}

Table 1: Properties of traps in CVD diamond. $\sigma$ is the capture cross-section for charge carriers.

Within CVD diamond, the main source of defect is thought to arise from nitrogen interstitials from the atmosphere [13][16][17]. Traps also exist due to the grain boundaries of the lattice crystals formed during the growth process [17] and are particularly important when considering polycrystalline diamond, where multiple grain boundaries exist [13].

However, there seems to be some variation among the literature, which may be down to the variation of techniques used to identify the traps ( $\mathrm{TSC}^{1}$, PICTS $^{2}$, alpha particle response measurements, etc [15]) or due to the continual development in the quality of diamond growth (i.e. samples vary) [13], but there is a general consensus of shallow traps around $0.3-04 \mathrm{eV}$ and deep traps around 1.1-1.3 eV.

Lebedev [12] discusses the main trap energy levels for $\mathrm{SiC}$ in great detail, with common impurities and defects being at much shallower levels than for D (relative to the Conduction and Valence band, the trap energy levels are in the region of $-0.97 \mathrm{eV}$ and $+0.63 \mathrm{eV}$ respectively). Consequently the charge carrier detrapping time would be expected to be less in $\mathrm{SiC}$ than that observed in D. Once again there is variation in the literature as to the origin or type of traps, but a significant volume of work discusses the so-called Z1 defect from 0.63 to $0.68 \mathrm{eV}$ which seems to be common across all variations of $\mathrm{SiC}$.

\footnotetext{
${ }^{1}$ Thermally stimulated current technique

${ }^{2}$ Photo-induced current transient spectroscopy
} 
The concentration of traps and the length of time carriers are trapped in them is an important factor in the quality of a detector. If any of the charge carriers are lost or delayed, so that they do not fully induce their charge during the integration time of the system, then the induced charge on the electrodes will be incomplete and the resultant signal pulse reduced. Furthermore, the emissions of detrapped electrons and holes outside of the integration time of their associated event, may also add to the noise of the system [11].

The ability of a detector to fully complete the collection of created charge is a measurable quantity simply given by its charge collection efficiency $(C C E)$,

$$
C C E=\frac{Q_{c}}{Q_{0}}
$$

where $Q_{c}$ and $Q_{0}$ are the amount of charge collected and created respectively.

As the velocity of the charge carrier is proportional to the applied electric field $(\vec{E})$ and the charge carrier drift mobility $(\mu)[1]$, the $C C E$ is dependent upon not only the material, but also the applied electric field strength, as small fields lead to a slow movement of charge carriers and therefore increased probability of trapping or recombination, where as larger fields reduce the probability. As such it is more convenient to consider the mobility lifetime product of electrons $\left(\mu_{e} \tau_{e}\right)$ and holes $\left(\mu_{h} \tau_{h}\right)$ as a characteristic property of that material.

The mobility lifetime product for each respective charge carrier is a combination of the $\mu$ within the material and the mean carrier lifetime $(\tau)$, that being the average period of time the created carriers exist free and hence can travel before they are trapped. It is therefore possible to define the movement of the charge carriers in terms of the mean drift length $(\lambda)$,

$$
\begin{aligned}
\lambda_{e} & =\mu_{e} \tau_{e} E \\
\lambda_{h} & =\mu_{h} \tau_{h} E
\end{aligned}
$$

as essentially $v=\lambda / \tau$, if it is assumed that $v$ is constant throughout the material. This quantity, which is strongly dependent upon $\mu \tau$, is extremely important in determining the quality of a material as $\lambda$ needs to be equal to or greater than the sensitive region of the detector in order to approach $100 \%$ $C C E$, i.e. the charge carriers must be able to travel the distance between electrodes in order to be fully collected by them. 
As proposed by Hecht [18], for the simple case of charge carriers passing through a parallel electrode geometry, $\lambda$ can be determined via Equation 4 [1],

$$
C C E=\frac{\lambda_{e}}{x}\left[1-\exp \left(\frac{x_{i}-x}{\lambda_{e}}\right)\right]+\frac{\lambda_{h}}{x}\left[1-\exp \left(-\frac{x_{i}}{\lambda_{h}}\right)\right]
$$

where $x$ is the detector thickness and $x_{i}$ is the radiation interaction location measure from the cathode. In Equation 4 it is assumed that the electric field is constant throughout the detector, which is a reasonable assumption for this simplified geometry.

In situations where the interaction depth of the radiation incident upon the anode is small compared to the thickness of the detector $\left(x_{i} \ll x\right)$, this expression can be simplified to,

$$
C C E=\frac{\lambda}{x}\left[1-\exp \left(\frac{x}{\lambda}\right)\right]
$$

and as such the mobility lifetime product of the electrons $\left(\mu_{e} \tau_{e}\right)$ and holes $\left(\mu_{h} \tau_{h}\right)$ can be independently determined by irradiating the anode or cathode respectively.

\section{Neutron Interactions}

As can be seen from Figures $1 \mathrm{a}$ and $1 \mathrm{~b}$ the most common fast neutron reaction in $\mathrm{Si}$ and $\mathrm{C}$ is elastic recoil scattering where the incident neutron (n) transfers a portion of its kinetic energy to the absorbing material through direct collisions.

Elastic interactions result in a change of direction and energy for the incident neutron, making it a scattered neutron (n'), as well as a gain of energy and momentum for the nucleus which recoils. If the energy transferred to this nucleus is such that its velocity is greater than that of its orbital electrons, it will lose those electrons and move through the medium as a heavy charged particle. The $Q$ value (the amount of energy required or released for the reaction) for this interaction is 0 (neglecting the binding energy of the nucleus in the lattice, which is a few tens of $\mathrm{eV}$ ) as conservation laws dictate that the energy of the reaction products (recoil nucleus and scattered neutron) must be the same as the incident particle.

From [1], when a neutron with nonrelativistic energy $\left(E_{n} \ll 939 \mathrm{MeV}\right)$ is incident upon a target nucleus, conservation of momentum and energy, the recoil angle $(\theta)$ of the nucleus with mass number $A$ is given by, 


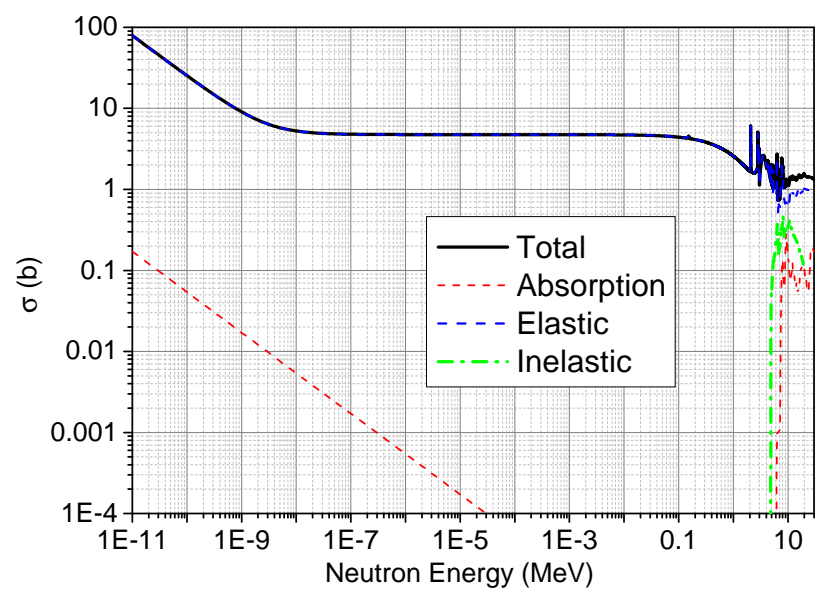

(a) Carbon-12

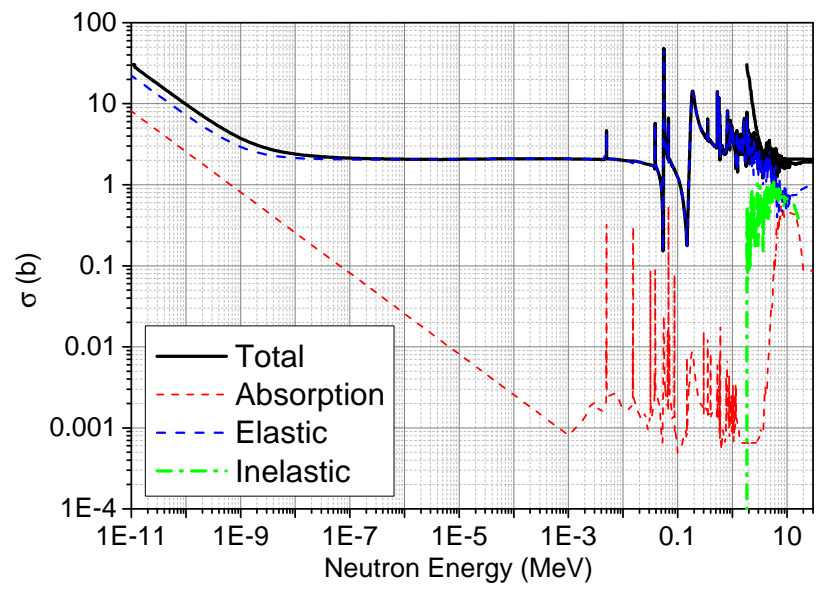

(b) Silicon-28

Figure 1: Elastic, absorption and total neutron cross sections $(\sigma)$ against neutron energy $\left(E_{n}\right)$, taken from NIST neutron scattering lengths and cross sections database [19], KAERI ENDF Cross section data [20] and BNL National Nuclear Data Center [21]. 


$$
\cos \theta=\sqrt{\frac{1-\cos \Theta}{2}}
$$

\section{Figure 2.}

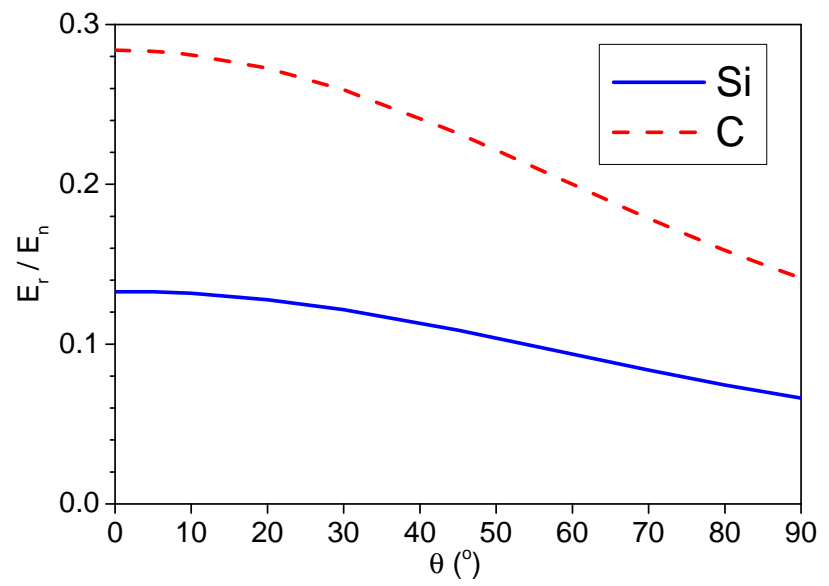

Figure 2: Energy transfer ratio $\left(E_{r} / E_{n}\right)$ against angle of recoil nucleus.

where $\Theta$ is the scattering angle of the incident neutron and it is assumed that the target nuclei are at rest. Therefore the recoil energy $\left(E_{r}\right)$ is given by Equation 7 [1],

$$
E_{r}=\frac{4 A}{(1+A)^{2}}\left(\cos ^{2} \theta\right) E_{n}
$$

From Equation 7 it is clear that the maximum transfer of energy occurs when the scattering angle of the incident neutron is $180^{\circ}$ or the recoil angle of the nucleus is $0^{\circ}$ (i.e. a head on collision). The neutron interactions will occur at various incident angles thus producing recoil products over a range of angles, resulting in a lower average energy transfer, as demonstrated in

The transfer of energy is further maximised when the mass of the absorber nuclei is small. For example the maximum conversion ratio $\left(E_{r} / E_{n}\right)$ for hydrogen is 1.0 , where as for carbon and silicon it is 0.28 and 0.13 respectively.

For this reason, low $Z$ materials are often used as fast neutron absorbers, of which hydrogen, often in the form of water $\left(\mathrm{H}_{2} \mathrm{O}\right)$ or plastic $\left(-\mathrm{CH}_{2^{-}}\right)$, is by far the most popular. These low $Z$ materials can be used for direct detection 
(such as proton recoil detectors) or for conversion detection (hydrogenous convertor layer producing detectable recoil protons), although conversion based detectors can suffer from low intrinsic efficiencies limited by a combination of successive interaction probabilities and self-attenuation [2].

At higher neutron energies $(>1 \mathrm{MeV})$ specific nuclear absorption reactions may begin to contribute more to the overall neutron interaction method, as demonstrated in Table 2.

For mono-energetic neutron sources the emission energy is very well defined, with some facilities capable of energies determined to be within $\pm 1.5 \%$. For radionuclide sources however, there is a very wide range of neutron energies emitted due to the complex nature of the interactions occurring. With Cf-252 and AmBe radioisotope neutron sources, for example, despite having an average energy of $2.1 \mathrm{MeV}$ and $4.1 \mathrm{MeV}$, neutrons can be emitted with energies ranging from, in principle, thermal energies $(<0.025 \mathrm{eV})$ up to $15 \mathrm{MeV}$ and $11 \mathrm{MeV}$ respectively [23].

Furthermore, gamma and X-ray emissions are present due to interactions within the radionuclide, surrounding material or due to the radioactive decay itself, as demonstrated in Figure 3. However, these plots do not take into consideration scattering or interactions from the test environment, which would likely change the neutron spectral distribution and add to the nonneutron radiation environment present (e.g. ionising photons).

\section{Gamma Interactions}

For the predominant processes by which X-ray and gamma-ray ionising photons interact with matter (Photoelectric Absorption, Compton Scattering and Pair Production), the probability of each process is proportional to both the energy of the incident photons $\left(E_{\gamma}\right)$ and the atomic number $(Z)$ of the material through which they are travelling. Consequently, low $Z$ material aids fast neutron interactions while also minimises gamma interactions, aiding discrimination between the two radiation types.

At higher energies, usually in the order of $>2 \mathrm{MeV}$, direct nuclear reactions also start to become significant, leading to the emission of highly ionising, heavy charged particles or neutrons, [25][26]. However, the ionising photon energies used within this investigation fall under these limits and as such these reactions will not be discussed. 


\begin{tabular}{|c|c|c|c|c|}
\hline Reaction & $\begin{array}{l}\text { 14MeV Neutron } \\
\text { Cross-section } \\
\text { (b) }\end{array}$ & $\begin{array}{c}\text { Reaction Energy } \\
\text { Threshold } \\
(\mathrm{MeV})\end{array}$ & $\begin{array}{l}Q \text {-value } \\
(\mathrm{MeV})\end{array}$ & $\begin{array}{c}\text { Branching } \\
(\mathrm{MeV})\end{array}$ \\
\hline${ }^{12} \mathrm{C}\left(\mathrm{n}, \mathrm{n}^{\prime}\right){ }^{12} \mathrm{C}$ & 0.2106 & - & 0 & - \\
\hline${ }^{12} \mathrm{C}\left(\mathrm{n}, \mathrm{n}^{\prime}\right){ }^{12} \mathrm{C}^{+2}$ & 0.2106 & 4.8088 & -4.4389 & - \\
\hline${ }^{12} \mathrm{C}(\mathrm{n}, \alpha){ }^{9} \mathrm{Be}$ & 0.0623 & 6.4196 & -5.7012 & $\begin{array}{l}\alpha_{0}=8.298 \\
\alpha_{1}=6.614 \\
\alpha_{2}=5.869\end{array}$ \\
\hline${ }^{12} \mathrm{C}\left(\mathrm{n}, \mathrm{n}^{\prime}\right) 3 \alpha$ & 0.2000 & 8.4286 & -7.3666 & - \\
\hline${ }^{12} \mathrm{C}(\mathrm{n}, \mathrm{p}){ }^{12} \mathrm{~B}$ & 0.0002 & 13.7401 & -12.5865 & $\begin{array}{l}\alpha_{0}=1.413 \\
\alpha_{1}=0.460\end{array}$ \\
\hline${ }^{28} \mathrm{Si}(\mathrm{n}, \mathrm{n})^{\prime 28} \mathrm{Si}$ & 0.1244 & - & 0 & - \\
\hline${ }^{28} \mathrm{Si}\left(\mathrm{n}, \mathrm{n}^{\prime}\right){ }^{28} \mathrm{Si}^{+2}$ & 0.1244 & 1.8425 & -1.7790 & - \\
\hline${ }^{28} \mathrm{Si}(\mathrm{n}, \alpha){ }^{25} \mathrm{Mg}$ & 0.1780 & 2.7653 & -2.6537 & $\begin{aligned} \alpha_{0} & =11.34 \\
\alpha_{1} & =10.76 \\
\alpha_{2} & =10.37 \\
\alpha_{3} & =9.734 \\
\alpha_{4} & =9.381 \\
\alpha_{5} & =8.544 \\
\alpha_{6} & =7.941 \\
\alpha_{7} & =7.932 \\
\alpha_{8} & =7.438 \\
\alpha_{9} & =7.375 \\
\alpha_{10} & =7.286 \\
\alpha_{11} & =7.069 \\
\alpha_{12} & =6.986\end{aligned}$ \\
\hline${ }^{28} \mathrm{Si}(\mathrm{n}, \mathrm{p})^{28} \mathrm{Al}$ & 0.2794 & 4.0042 & -3.8599 & $\begin{array}{l}\alpha_{0}=10.14 \\
\alpha_{1}=10.11 \\
\alpha_{2}=9.167 \\
\alpha_{3}=9.126 \\
\alpha_{4}=8.767\end{array}$ \\
\hline
\end{tabular}

Table 2: Fast neutron nuclear reactions within $\mathrm{Si}$ and $\mathrm{C}$, taken from [20][21][22]. Values $\alpha_{0}, \alpha_{1} \ldots \alpha_{n}$ represent the ground to excited states of emission. 


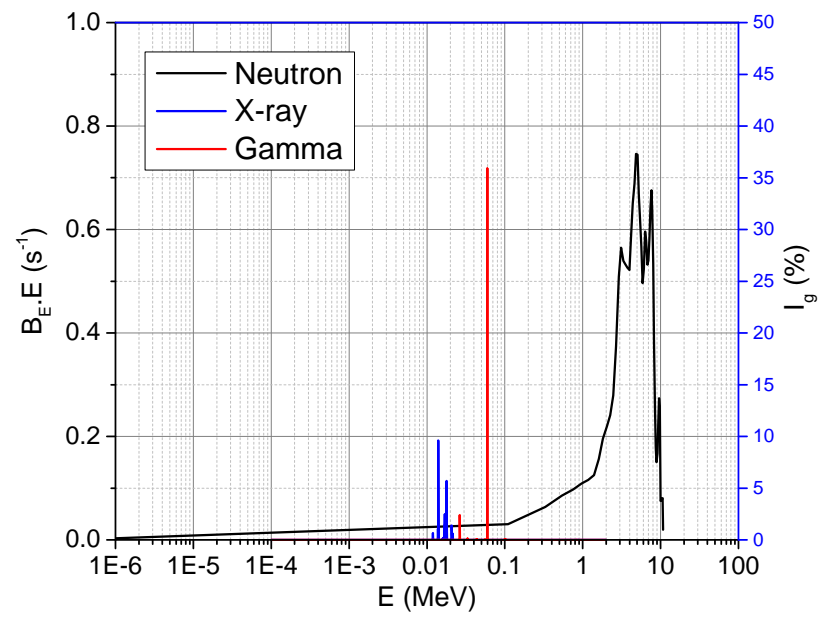

Figure 3: Theoretical AmBe neutron energy spectrum lethargy distribution $\left(B_{E} \cdot E\right)[23]$ with Am-241 X-ray and gamma emission intensities $\left(I_{g}\right)[24]$ against energy $(E)$.

\section{Experimental Methods}

The single crystal diamond detector (D-SC) was fabricated from high purity, electronic grade, single crystal diamond grown by Element Six Ltd ${ }^{\odot}$ using the chemical vapour deposition (CVD) technique [27]. $4 \mathrm{~mm} \times 4 \mathrm{~mm}$ platinum contacts were sputter deposited onto the $5 \times 5 \mathrm{~mm}$ by $500 \mu \mathrm{m}$ thick sample at the University of Surrey, following a thorough cleaning process of boiling sulphuric acid and potassium nitrate, then de-ionised water, acetone and isopropanol respectively. The detector was mounted on a ceramic board and bonded with $24 \mu \mathrm{m}$ gold wire. During testing, unless otherwise stated, this detector was operated at $-200 \mathrm{~V}$ bias.

The polycrystalline diamond detector (D-PC) was fabricated from $20 \times 20 \mathrm{~mm}$ by $300 \mu \mathrm{m}$ thick polycrystalline CVD diamond from Diamond Detectors Limited ${ }^{\odot}$ [28]. This material was thoroughly cleaned prior to contact fabrication using aqua regia, acetone and isopropanol respectively. $\varnothing 6.5 \mathrm{~mm}$ gold metal contacts were sputtered deposited at the University of Surrey and the completed detector mounted on a bespoke, low impedance $(50 \Omega)$ printed circuit board and bonded with $24 \mu \mathrm{m}$ gold wire. The detector was operated at $-400 \mathrm{~V}$ bias during the investigation.

The semi-insulating $\mathrm{SiC}$ detector (SiC-SI) was fabricated from $\mathrm{Cree}^{\odot} 4 \mathrm{H}-$ 
$\mathrm{SiC}$ material [8]. The SiC-SI was $7 \times 7 \mathrm{~mm}$ by $360 \mu \mathrm{m}$ thick and had a net doping of less than $10^{5} \mathrm{~cm}^{-3}$. The $5 \times 5 \mathrm{~mm}$ contacts consisted of $\mathrm{Ti} / \mathrm{Pt} / \mathrm{Au}$ Schottky contacts and $\mathrm{Ni} / \mathrm{Au}$ ohmic contacts, applied via a combination of photo-lithography and vacuum-deposition. The detector was mounted upon a printed circuit board at the University of Surrey and operated at $-400 \mathrm{~V}$.

For all the testing conducted the detectors were mounted within either a light sealed diecast metal test box or vacuum cryostat connected to ORTEC 142A charge sensitive preamplifiers; ORTEC 570 or 572 shaping amplifiers; an ORTEC 710 quad-bias supply; and ORTEC Easy-MCA with associated Maestro software.

All alpha spectra tests were conducted with a $3.7 \mathrm{kBq}$ Am-241 source inside a vacuum chamber at a pressure of $8 \times 10^{-2}$ mbar. Alpha polarisation testing was conducted in air with a $60 \mathrm{kBq}$ Am-241 source.

Neutron testing was conducted in air, at AWE and Thermo Fisher Scientific ${ }^{\odot}$ (Beenham) for radionuclide sources, as well as the National Physical Laboratory $\left(\mathrm{NPL}^{\odot}\right)$ for radionuclide and mono-energetic neutron sources. Where possible, these tests were conducted in fields greater then $4 \mathrm{mSv} / \mathrm{h}$ and irradiated for times exceeding 2 hours in order to obtain reasonable counting statistics in all channels.

Between each radiation exposure the detectors were exposed to at least 15 minutes of room ambient light while at $0 \mathrm{~V}$ bias in order to de-polarise the detector.

Energy calibration of the detectors was conducted using pulser-capacitor calibrations as described by Siegbahn [29].

\section{Results}

All of the detectors under test showed distinguishable alpha spectra as demonstrated in Figure 4, with the energy resolution above background noise improving as the bias increases. The SiC-SI and D-SC detectors were also able to demonstrate suitable resolution in order to distinguish the trailing edge of the alpha source used, an artefact from the source not being externally plated with Am-241 material and resulting in a portion of lower energy alpha particles emissions due to the subsequent interactions with the emission window.

The effect of applied bias on the charge collection efficiency $(C C E)$ and intrinsic efficiency (count rate/incident radiation flux) has been depicted in Figure 5. The D-SC shows the best alpha spectroscopy performance with 


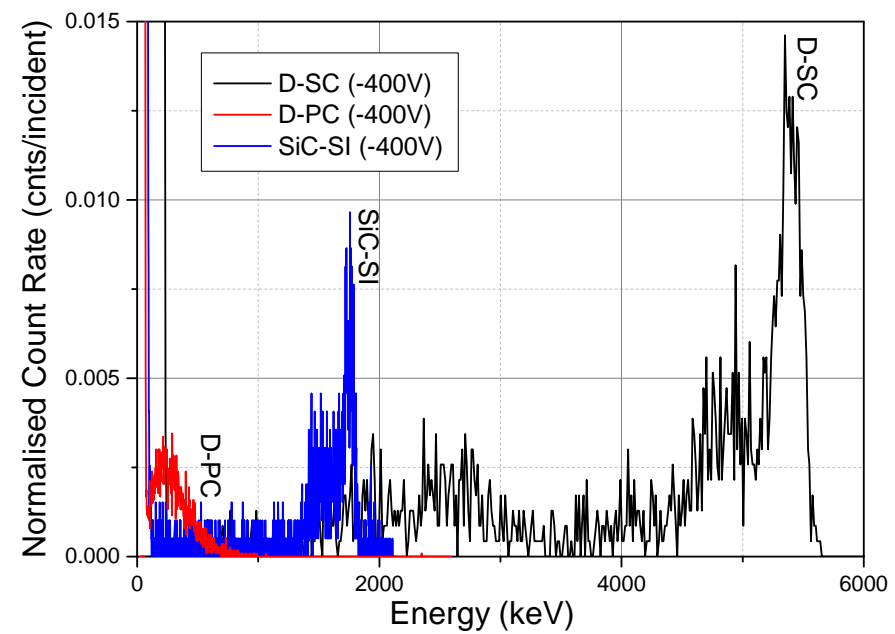

Figure 4: Count rate, normalised to incident flux, against energy. All detectors were tested at $8 \times 10^{-2}$ mbar with a $3 \mathrm{kBq} A m-241$ alpha source. The different peak positions are a direct realist of the $C C E$ of the detector.

$100 \% C C E$ and intrinsic counting efficiency from around -200V. This detector also shows $100 \% C C E$ above $+200 \mathrm{~V}$, but the intrinsic efficiency is limited to $60-70 \%$ due to a slightly higher noise threshold level during the positive bias testing because of poor electrical continuity.

Using the simplified version of the Hecht equation (Equation 5) the mobility lifetime products of the electrons $\left(\mu_{e} \tau_{e}\right)$ and holes $\left(\mu_{h} \tau_{h}\right)$ in D-SC were determined to be $(5.6 \pm 0.1) \times 10^{-5} \mathrm{~cm}^{2} \mathrm{~V}^{-1}$ and $(6.1 \pm 0.3) \times 10^{-5} \mathrm{~cm}^{2} \mathrm{~V}^{-1}$ respectively. This is in good agreement with the results presented by AbdelRahman [30], with deviations related to the peak position selection and polarisation effects, which make the analysis sensitive to the source activity and measurement duration.

The SiC-SI detector shows an increase in intrinsic efficiency with negative bias up to a maximum of $100 \%$, despite a maximum $C C E$ of $35 \%$ over the same range. The minimum depletion width suggested by capacitance measurements $(94 \mu \mathrm{m})$ is more than sufficient relative to the alpha penetra-

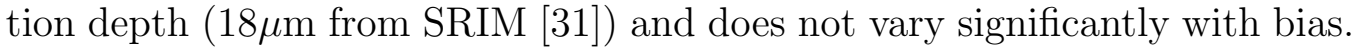
Therefore the low $C C E$ and high intrinsic efficiency $\left(\epsilon_{i}\right)$ is likely a result of the incomplete charge collection due to defect or impurity traps within the material. 
In contrast to these results, the work conducted by Ruddy et al. on similar detector material [8] showed a $C C E$ of $27 \%$ at a maximum bias $400 \mathrm{~V}$. It was proposed in that work that the maximum bias was a limit of the package the detector was mounted in. It should however be noted that during this investigation the SiC-SI detector achieved $\approx 60 \% C C E$ at $-900 \mathrm{~V}$ during characterisation.

The calculated mobility lifetime products were determined to be $(3.77 \pm$ $0.01) \times 10^{-6} \mathrm{~cm}^{2} \mathrm{~V}^{-1}$ and $(0.34 \pm 0.01) \times 10^{-6} \mathrm{~cm}^{2} \mathrm{~V}^{-1}$ for the electrons and holes respectively. The ratio of the mobility lifetime products $\left(\mu_{e} \tau_{e} / \mu_{h} \tau_{h}=\right.$ 11 ) is in good agreement with the expected $\mathrm{SiC}$ electron-hole mobility ratio (reported $\mu_{e} / \mu_{h}$ range from 9 to 16 [32]).

Finally the D-PC detector demonstrated a very small charge collection efficiency of approximately $4 \%$ and only 30-35\% intrinsic efficiency for both positive and negative bias, corresponding to mobility lifetime products of $(8.0 \pm 0.9) \times 10^{-8} \mathrm{~cm}^{2} \mathrm{~V}^{-1}$ and $(6.7 \pm 0.7) \times 10^{-8} \mathrm{~cm}^{2} \mathrm{~V}^{-1}$ for electrons and holes respectively.

Figure 6a shows the effect on the D-SC AmBe neutron spectrum as the amount of lead $(Z=82)$ between the source and detector increases. Figure $6 \mathrm{~b}$ also shows the experimentally determined attenuation (i.e. observed reduction in intrinsic count rate) of the AmBe neutrons as a function of lead thickness. This has been plotted against the theoretical attenuation of Co-60 gammas [33] (important for fission created gammas) and Am-241 gammas [33] (one of the main gamma emitters from the AmBe) to demonstrate that even a few $\mathrm{mm}$ of lead will fully attenuate the main gamma emissions from the AmBe source without seriously affecting the neutron spectra $(<1 \%$ attenuation) recorded by the detectors. In fact, the rate of attenuation is still greater for Co-60 than it is for AmBe neutrons, with $11 \mathrm{~mm}$ of lead attenuating the AmBe signal by $\approx 28 \%$ and the Co- 60 by $\approx 51 \%$.

It is worth noting that the data in Figures $6 \mathrm{a}$ and $6 \mathrm{~b}$ is important when considering how to optimise these detectors for practical applications as it essentially shows that by reducing the X-ray or gamma ray influence with high $Z$ filtration, any cross-sensitivity threshold can be reduced, thus increasing the neutron/gamma count rate ratio. Although some neutron attenuation will occur as a result of the filtration, it will not be to the same extent as the X-rays or gammas, subsequently leading to a better neutron-gamma cross-sensitivity ratio (more neutrons per gamma).

From this work it is with a high level of confidence that the data presented in Figure 7 demonstrates that the detectors are directly detecting fast 


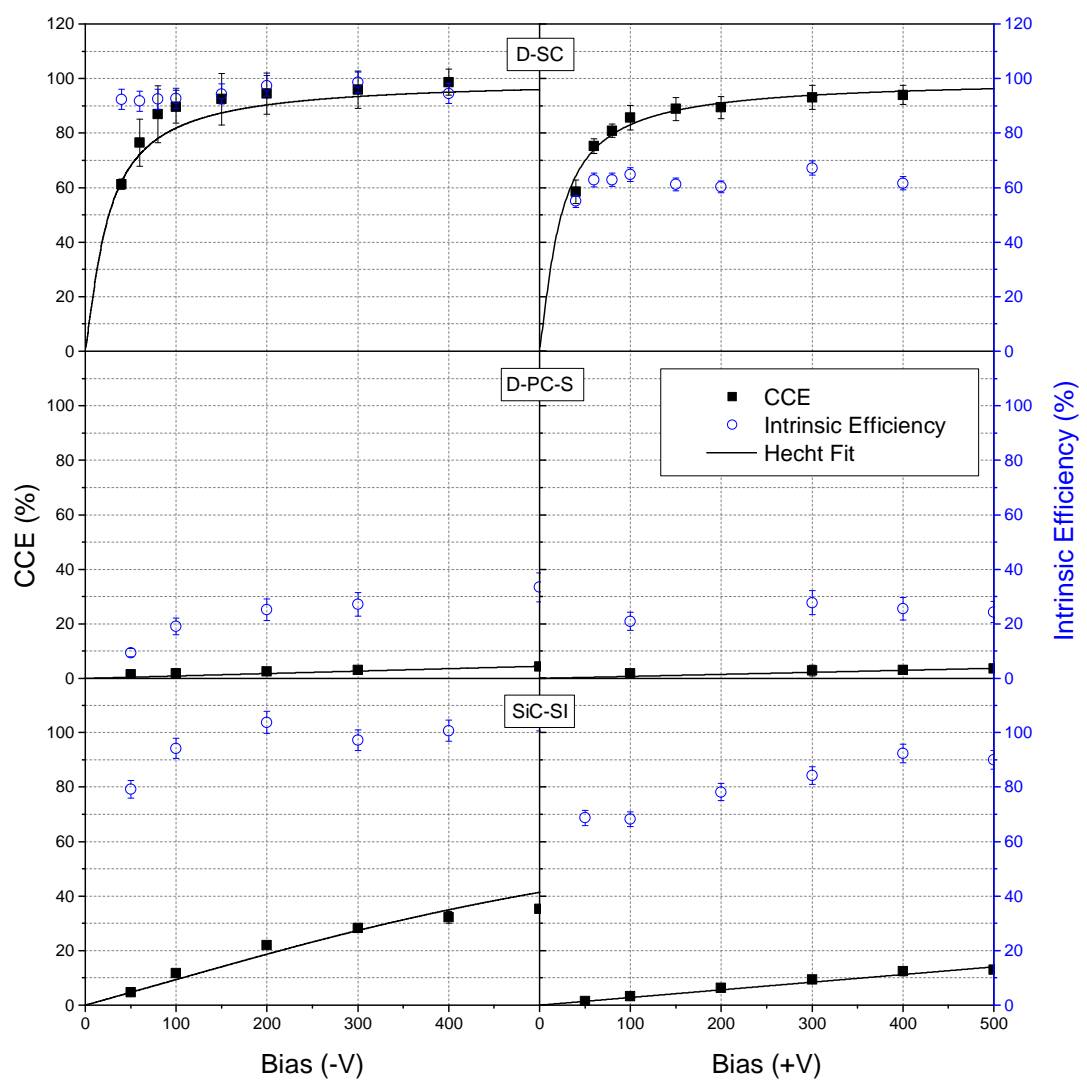

Figure 5: Charge collection efficiency (CCE) and intrinsic efficiency against bias for D-SC, D-PC and SiC-SI. For detectors with suitable geometry and electric field profile assumptions, a simplified Hecht fit has been plotted. 


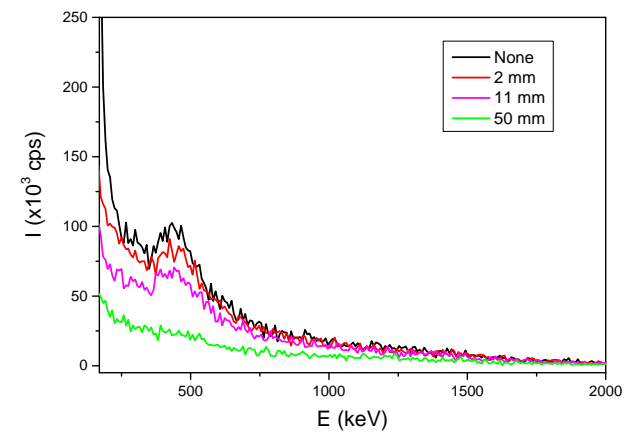

(a) Count rate $(I)$ against energy $(E)$ for D-SC detector irradiated with an AmBe neutron source $(\approx 4 \mathrm{mSv} / \mathrm{h})$ as a function of lead $(\mathrm{Pb})$ shielding thickness.

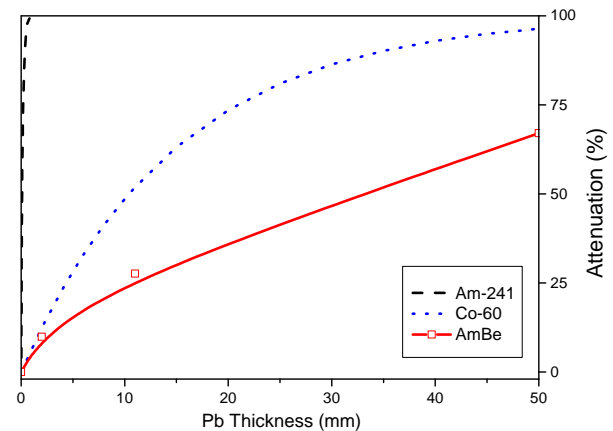

(b) The attenuation of Am-241 gammas [33], Co-60 gammas [33] and AmBe neutrons (experimental data) as a function of lead $(\mathrm{Pb})$ thickness.

Figure 6: The effect of lead on the gamma and neutron detection performance of D-SC detector at -400V.

neutrons despite low charge collection efficiency and / or polarisation issues. The Cf-252 and mono-energetic neutron data in particular emphasises this as the gamma emissions from these sources are both low intensity and low energy. Furthermore, by comparing the end point energies of each spectrum it is clear that a simple energy threshold level could be used to discriminate neutron energies.

The results presented in Figure 7 have been normalised to the incident flux of the neutron radiation. For the mono-energetic sources, the incident flux primarily consists of the main neutron energy and therefore the position on the y-axis in the graphs presented is a good representation of the sensitivity of the detectors to that radiation type. For radionuclide sources however, this method of normalisation is less accurate as there is a wide spectrum of incident neutron energies, each of which have different interaction probabilities. It does however give a reasonable indication of the relative sensitivity over the entire energy range.

Data points with the suffix $L G$ represent data taken with a lower gain calibration setting. This was required for radiation sources where the maximum energy was larger than the calibrated energy range of the detectors. Aside from allowing the higher energy spectra to be acquired, it also changed the energy binning leading to a perceived increase in sensitivity over some regions, particularly those with high counting statistics (i.e. lower energy channels). 
For D-SC this led to a change in the energy bin width to $9.5 \mathrm{keV} /$ channel from $18.8 \mathrm{keV} /$ channel; $4.7 \mathrm{keV} /$ channel from $10.9 \mathrm{keV} /$ channel for D-PC; and $2.6 \mathrm{keV} /$ channel from $6.1 \mathrm{keV} /$ channel for SiC-SI. As such, both the standard and $L G$ data have been shown in the graphs in order to give a better indication over the entire neutron spectra energy range.

Analysis of Figure 7 shows that the D-SC detector provides the best overall neutron performance, with the observed end point energies corresponding to the expected maximum energies for all the neutron sources tested and the count rate per incident neutron is higher relative to the other detectors under test. This seems reasonable when considering the high neutron energy transfer ratio (Figure 2), larger thickness and the $C C E$ of the detector. The D-SC performance is also in excellent agreement with the work of Pillon et al. [9] with clear nuclear reaction and recoil features.

The D-PC demonstrates reasonable neutron detection characteristics despite the very low $C C E$, highlighting the benefit of carbon based detectors. In fact the end point energies for this detector seem to exceed expectations, as they are significantly higher than would be expected for a detector at around 3-4\% CCE which is likely a result of both the high energy transfer ratio $\left(E_{r} / E_{n}\right)$ of carbon and the neutron interactions being more uniform across the detector relative to alpha particles (i.e. no localised trapping). However, there are no clear nuclear reactions or recoil features within the spectra, which will be a direct result of the low peak resolution in the detector.

The $\mathrm{SiC}-\mathrm{SI}$ spectrum presented for AmBe is similar to that presented by Bryant [34] and the features correspond to the simulated response in the same paper. The endpoint energies are also comparable to the expected maximum energies for all the neutron sources tested when taking into account the $C C E$ of the material ( $\approx 32 \%$ ) although the overall sensitivity is not as high as the diamond detectors, likely related to the lower energy conversion efficiency of the Si atoms within the detector.

As can be seen in Figure 8 the performance of these detectors is maintained over a fairly wide dynamic range of neutron fluxes. This shows that the observations are indicative of direct neutron detection as the count rate increases linearly with the radiation dose received, demonstrating that the chosen low energy threshold is effectively discriminating electronic noise and that the counts observed are all due to real events.

All the detectors demonstrated some issues with stability during irradiation, namely changes to the acquired spectrum and / or count rate with time. 


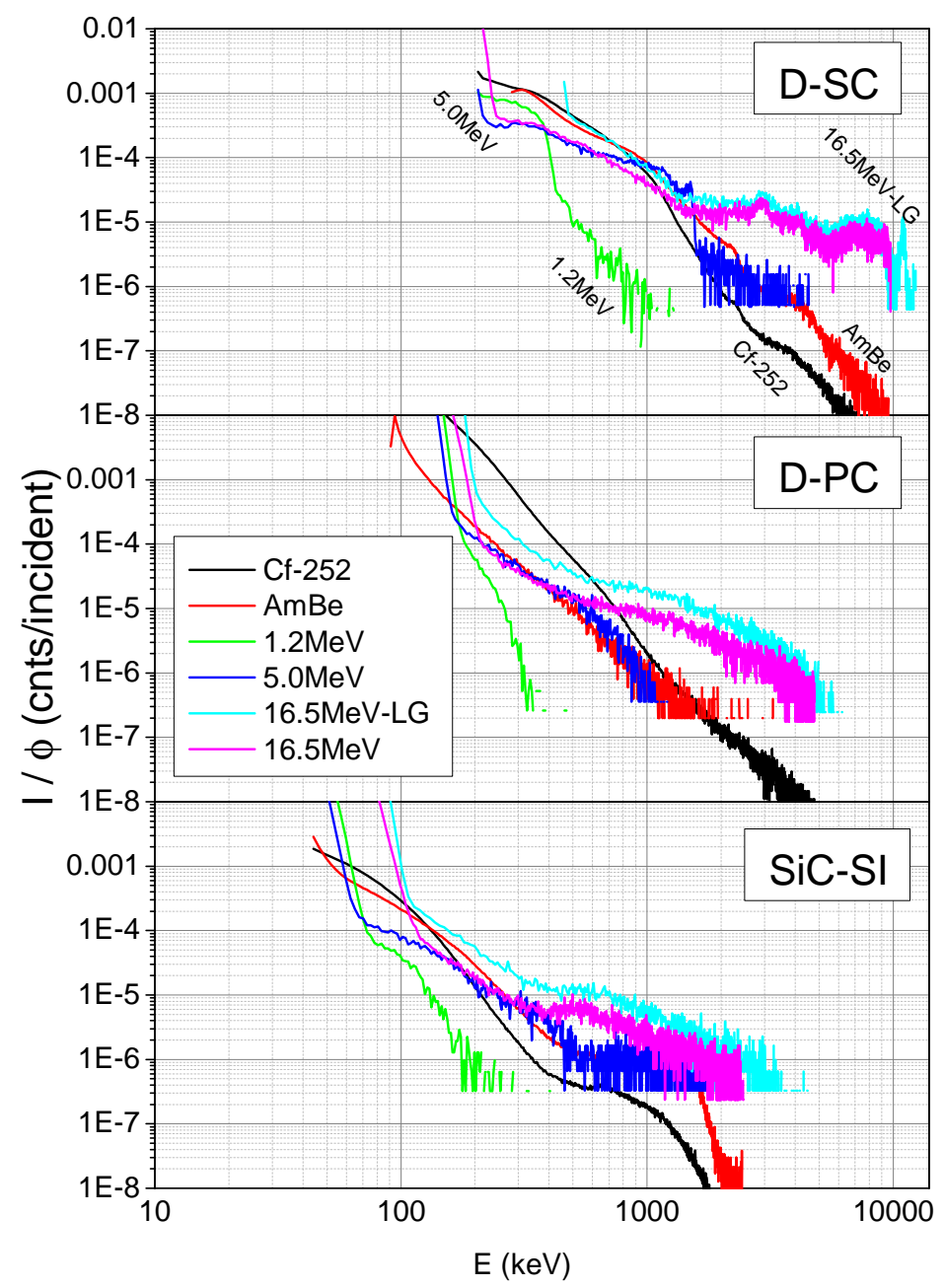

Figure 7: Count rate, normalised to incident flux $(I / \phi)$, against energy $(E)$. All exposures were conducted at NPL ${ }^{\odot}$. $L G$ represents a lower gain calibration setting used during test so that the high energy points were visible in the spectrum. 


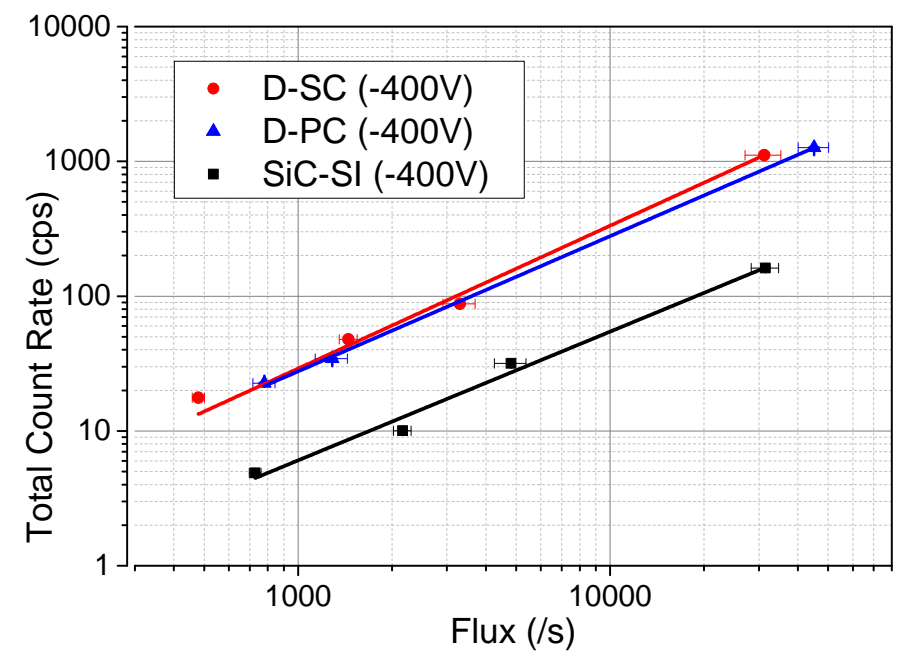

Figure 8: Total count rate above a given threshold against incident radiation flux for Cf-252 neutron radiation. Linear fit displayed with log-log gradients of 1.004, 1.000, 0.955 for D-SC, D-PC and SiC-SI respectively.

This so-called polarisation effect is dependent upon the concentration of ionisation within the detectors, with highly ionising particles (such as alphas) producing very quick polarisation effects, as demonstrated in Figure 9. This is because these particles tend to create a large amount of charge carriers at shallow depths within the detector $(\approx 17 \mu \mathrm{m}$ for diamond and $\approx 18 \mu \mathrm{m}$ for $\mathrm{SiC}$ [31]) resulting in a high trapping rate over a small region[5][6][7]. This leads to the creation of a localised space charge barrier close to the electrode, through which further electrons and holes must pass to be fully collected.

For SiC-SI and D-PC, over the time frame tested, this leads to a potentially paralyzable effect within the detector as the spectrum moves into the noise region and no further pulses can be registered. This effect highlights the difference between the mobility lifetime products of the detectors, with $\mathrm{D}-\mathrm{PC}$ having the lowest $\mu \tau$ product and the highest polarisation rate as charge trapping is very likely.

D-SC on the other hand had a relatively good $\mu \tau$ product, resulting in a low polarisation rate and even recovery of the count rate over longer time periods. This count rate recovery is a direct observation of priming whereby the traps within the material are steadily filled with the created charge carri- 
ers until they reach saturation point, after which a stable field, and therefore spectrum, is maintained [6]. The relatively good mobility lifetime product of this material ensures that the subsequently created electrons and holes are capable of traversing the detector even with an altered space charge region. Therefore, as the charge carrier creation rate is maintained for a constant irradiation field, the count rate steadily returns to the original value as less charge carriers are trapped and lost from the counting system.

For neutrons the charge carriers are created, on average, more uniformly throughout the entire detector thickness, therefore the subsequent trapping would be distributed more evenly, diluting the overall space charge buildup and reducing the polarisation effect [35]. As such, it is expected that the polarisation rate for neutrons would be less than alphas, as observed in Figure 9.

In fact, for all the detectors under irradiation from $6 \mathrm{mSv} / \mathrm{h} \mathrm{Cf-252} \mathrm{neu-}$ trons, there was little or no polarisation observed ( $<5 \%$ variation over $80,000 \mathrm{~s})$ as the rate of de-trapping is greater than or equal to the rate of trapping (or charge carrier generation). However, as the dose rate increases the rate of polarisation increases for the low $C C E$ material $(<50 \%)$ as would be expected for increased charge carrier creation, but rarely discussed in the literature for neutrons.

Despite the onset of polarisation effects as the neutron dose rate is increased, operation is still possible, further demonstrated in Figure 8. For the D-PC detector in particular, the count rate stabilises during neutron induced polarisation, despite alpha induced polarisation leading to paralyzable affects. This demonstrates the point at which trapping and de-trapping is in equilibrium across the whole volume of the detector, where as the alpha data demonstrates the equilibrium at a shallow irradiation depth. As the neutron equilibrium is dependent upon the number of charge traps within the detector, potentially this uniform neutron polarisation effect could also be used in the future to characterise charge trap concentrations within detectors.

\section{Conclusion}

Work has been conducted to demonstrate that carbon based, wide band gap, semiconductor detectors with lower charge collection efficiency $(C C E)$, poor resolution and polarisation effect can fulfil requirements for fast neutron detection and spectroscopy over a fairly wide dynamic range. This demonstrates that suitable neutron detection performance and stability is possible 


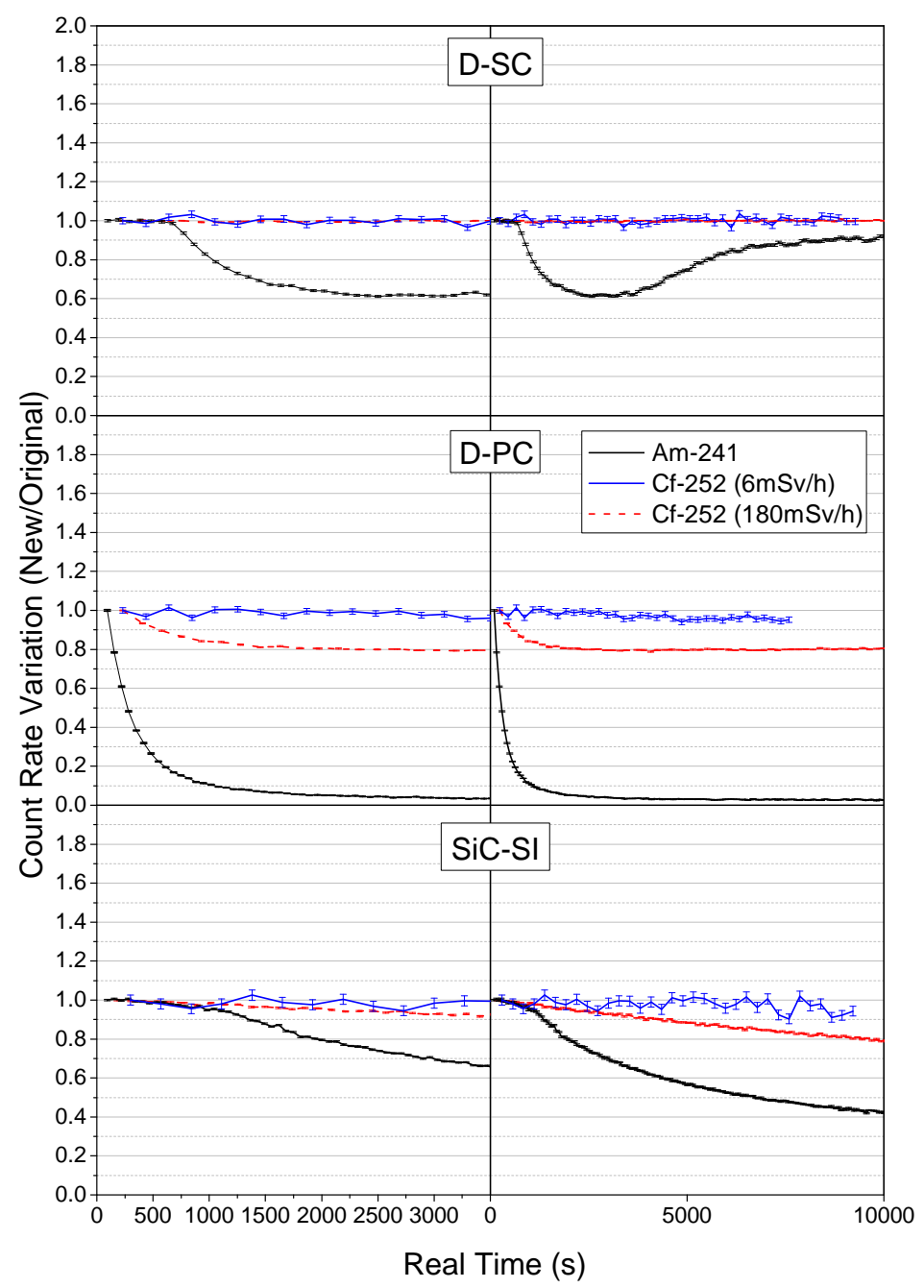

Figure 9: Count rate variation $\left(I / I_{0}\right)$ against time as a function of radiation type. Alpha source was a $60 \mathrm{kBq} A m-241$ at $8 \times 10^{2}$ mbar. Cf-252 data were taken at approximately $6 \mathrm{mSv} / \mathrm{h}$ and $180 \mathrm{mSv} / \mathrm{h}$. 
with lower quality polycrystalline diamond (D-PC) and semi-insulating silicon carbide (SiC-SI) detectors, increasing the potential options available for practical neutron applications where large areas/volumes are needed.

Single crystal diamond (D-SC) was shown to have $100 \%$ CCE, however, under the influence of alpha radiation it demonstrated the polarisation effect, by which the energy peak and count rate varied with irradiation time.

Paralyzable alpha induced polarisation effects were also observed in the D$\mathrm{PC}$ and SiC-SI detectors, as well as low $C C E$ ( $<5 \%$ and $<50 \%$ respectively).

Despite incomplete charge collection efficiency, poor energy resolution or polarisation issues, all these detectors did perform as fast neutron detectors over a fairly wide range of neutron fluxes, with less than $20 \%$ variation in the neutron count rate over the time tested.

Polarisation as a result of neutron irradiation was investigated within these detectors for neutron dose rates of $6 \mathrm{mSv} / \mathrm{h}$ and $180 \mathrm{mSv} / \mathrm{h}$, which, in the case of polycrystalline diamond and semi-insulating silicon carbide, is the first time it has been demonstrated. Overall it was shown that the polarisation rate for neutrons was less than alphas and could result in a stable detector, subject to the incident flux. This directly demonstrated that uniform charge carrier creation reduced the polarisation effect relative to the high-concentration charge carrier creation from shallow penetrating alpha particles.

Subsequently it can be concluded that despite, what is generally considered, unfavourable detection characteristics these detectors can be effective direct fast neutron detectors.

\section{Acknowledgements}

The authors would like to acknowledge the staff at the University of Surrey, NPL ${ }^{\odot}$, Thermo Fisher Scientific ${ }^{\odot}$ and AWE for there assistance with testing and analysis.

This work was funded by the United Kingdom Science Technology Funding Council (STFC grant number: ST/H003959/1) and in collaboration with AWE.

[1] G. F. Knoll, Radiation detection and measurement, 4th Edition, John Wiley, Hoboken, N.J, 2010, ISBN 97804701314800470131489.

[2] D. McGregor, M. Hammig, Y.-H. Yang, H. Gersch, R. Klann, Design considerations for thin film coated semiconductor thermal neutron detec- 
tors - I: basics regarding alpha particle emitting neutron reactive films, Nuclear Instruments and Methods in Physics Research Section A: Accelerators, Spectrometers, Detectors and Associated Equipment 500 (1-3) (2003) 272-308. doi:10.1016/S0168-9002(02)02078-8.

[3] A. Chynoweth, Behavior of Space Charge in Diamond Crystal Counters under Illumination. I, Physical Review 83 (2) (1951) 254.

[4] F. Franceschini, F. Ruddy, Silicon Carbide Neutron Detectors, in: R. Gerhardt (Ed.), Properties and Applications of Silicon Carbide, InTech, 2011.

[5] M. Rogalla, K. Runge, A. Söldner-Rembold, Particle detectors based on semi-insulating silicon carbide, Nuclear Physics B-Proceedings Supplements 78 (1) (1999) 516-520.

[6] L. Lan, O. Xiaoping, T. Xinjian, X. Liangbin, C. Na, L. Bing, Z. Xiaodong, Priming effect on a polycrystalline CVD diamond detector under 60-Co $\gamma$-rays irradiation, Nuclear Instruments and Methods in Physics Research Section A: Accelerators, Spectrometers, Detectors and Associated Equipment 672 (2012) 29-32. doi:10.1016/j.nima.2011.12.022.

[7] A. Lohstroh, P. J. Sellin, S. G. Wang, A. W. Davies, J. M. Parkin, Mapping of polarization and detrapping effects in synthetic single crystal chemical vapor deposited diamond by ion beam induced charge imaging, Journal of Applied Physics 101 (6) (2007) 063711.

[8] F. H. Ruddy, J. G. Seidel, R. W. Flammang, R. Singh, J. Schroeder, Development of radiation detectors based on semi-insulating silicon carbide, in: IEEE Nuclear Science Symposium Conference Record. NSS '08, 2008, pp. 449 -455. doi:10.1109/NSSMIC.2008.4775205.

[9] M. Pillon, M. Angelone, A. Krása, A. Plompen, P. Schillebeeckx, M. Sergi, Experimental response functions of a single-crystal diamond detector for $5-20.5 \mathrm{MeV}$ neutrons, Nuclear Instruments and Methods in Physics Research Section A: Accelerators, Spectrometers, Detectors and Associated Equipment 640 (1) (2011) 185-191. doi:10.1016/j.nima.2011.03.005. 
[10] A. Kumar, A. Singh, A. Kumar, A. Topkar, Fabrication and characterization of polycrystalline diamond detectors for fast neutron monitoring, Nuclear Instruments and Methods in Physics Research Section A: Accelerators, Spectrometers, Detectors and Associated Equipment 785 (2015) 55-60. doi:10.1016/j.nima.2015.02.044.

[11] G. Lutz, Semiconductor radiation detectors device physics, Springer, Berlin; New York, 2007, ISBN 9783540716792.

[12] A. A. Lebedev, Deep level centers in silicon carbide: A review, Semiconductors 33 (2) (1999) 107 to 130.

[13] S. G. Wang, P. Sellin, A. Lohstroh, Temperature-dependent hole detrapping for unprimed polycrystalline chemical vapor deposited diamond, Applied Physics Letters 88 (2) (2006) 023501.

[14] J. W. Glesener, Photoinduced current transient spectroscopy of boron doped diamond, Applied Physics Letters 63 (6) (1993) 767. doi:10.1063/1.109902.

[15] M. Bruzzi, D. Menichelli, S. Sciortino, L. Lombardi, Deep levels and trapping mechanisms in chemical vapor deposited diamond, Journal of Applied Physics 91 (9) (2002) 5765. doi:10.1063/1.1461891.

[16] P. Gonon, S. Prawer, D. Jamieson, Thermally stimulated currents in polycrystalline diamond films: Application to radiation dosimetry, Applied Physics Letters 70 (22) (1997) 2996. doi:10.1063/1.118768.

[17] M. H. Nazaré, Neves, A. J., Properties, Growth and Applications of Diamond, no. 26 in Emis Datareviews Series, INSPEC, 2001, ISBN 0 852967853.

[18] K. Hecht, Zum Mechanismus des lichtelektrischen Primssrstromes in isolierenden Kristallen, Zeitschrift for Physik 77 (3-4) (1932) 235-245. doi:10.1007/BF01338917.

[19] NIST, Neutron scattering lengths and cross sections, accessed 20-012013.

URL http://www.ncnr.nist.gov/resources/n-lengths/ 
[20] Korea Atomic Energy Research Institute, ENDF Cross Section Data, accessed 19-01-2013 (2000). URL http://atom.kaeri.re.kr/cgi-bin/endfplot.pl?j=\&d=\&f=

[21] Brookhaven National Laboratory, National Nuclear Data Center, accessed 19-01-2013.

URL http: //www.nndc. bnl.gov/

[22] F. Ruddy, A. Dulloo, J. Seidel, M. Das, S. Ryu, A. Agarwal, The fast neutron response of $4 \mathrm{~h}$ silicon carbide semiconductor radiation detectors, Nuclear Science, IEEE Transactions on 53 (3) (2006) 1666-1670.

[23] NCE/2, BS ISO 8529-1:2001 - Reference neutron radiations. Characteristics and methods of production, Neutronen-Referenzstrahlungen. Merkmale und Verfahren zur Erzeugung, International Organization for Standardization, 2001.

[24] S. Chu, L. Ekstrom, R. Firestone, B., The LUND/LBNL Nuclear Data Search, accessed 08-02-2013 (Feb. 1999).

[25] R. Runkle, D. Chichester, S. Thompson, Rattling nucleons: New developments in active interrogation of special nuclear material, Nuclear Instruments and Methods in Physics Research Section A: Accelerators, Spectrometers, Detectors and Associated Equipment 663 (2011) 75-95.

[26] T. Gozani, Fission Signatures for Nuclear Material Detection, IEEE Transactions on Nuclear Science 56 (3) (2009) 736 -741. doi:10.1109/TNS.2009.2015309.

[27] M. A. E. Abdel-Rahman, A. Lohstroh, P. J. Sellin, The effect of annealing on the X-ray induced photocurrent characteristics of CVD diamond radiation detectors with different electrical contacts, Physica Status Solidi a-Applications and Materials Science 208 (9) (2011) 2079-2086.

[28] F. Schirru, B. S. N. Singh, L. Scruton, M. A. Bentley, S. P. Fox, A. Lohstroh, P. J. Sellin, A. Banu, M. McCleskey, B. T. Roeder, E. Simmons, A. A. Alharbi, L. Trache, M. Freer, D. Parker, Development of large area polycrystalline diamond detectors for fast timing application of high-energy heavy-ion beams, Journal of Instrumentation 7 (05) (2012) P05005-P05005. doi:10.1088/1748-0221/7/05/P05005. 
[29] K. Siegbahn (Ed.), Alpha-, beta- and gamma-ray spectroscopy, NorthHolland Publ. [u.a.], Amsterdam, 1965, ISBN 0444106952.

[30] M. A. El-Rahman, The influence of electrode fabrication on induced Xray photocurrents in CVD diamond radiation detectors, Ph.D. thesis, University of Surrey (2011).

[31] J. Ziegler, SRIM \& TRIM, accessed 07-07-2015 (2013). URL http://www.srim.org/

[32] P. Sellin, J. Vaitkus, New materials for radiation hard semiconductor dectectors, Nuclear Instruments and Methods in Physics Research Section A: Accelerators, Spectrometers, Detectors and Associated Equipment 557 (2) (2006) 479-489. doi:10.1016/j.nima.2005.10.128.

[33] Rad Pro Calculator, accessed 09-02-2013.

URL http://www.radprocalculator.com/

[34] P. A. Bryant, A. Lohstroh, P. J. Sellin, Electrical Characteristics and Fast Neutron Response of Semi-Insulating Bulk Silicon Carbide, IEEE Transactions on Nuclear Science 60 (2) (2013) 1432-1435. doi:10.1109/TNS.2013.2243753.

[35] E.-K. Souw, R. Meilunas, Response of CVD diamond detectors to alpha radiation, Nuclear Instruments and Methods in Physics Research Section A: Accelerators, Spectrometers, Detectors and Associated Equipment 400 (1) (1997) 69-86. doi:10.1016/S0168-9002(97)00963-7. 